\title{
REPRESENTAÇÕES DO CUIDADO NO LIMIAR DA VIDA POR PROFISSIONAIS DE UMA UNIDADE DE TERAPIA INTENSIVA MÓVEL
}

Keyla Cristiane do Nascimento' ${ }^{1}$, Alacoque Lorenzini Erdmann ${ }^{2}$,

Eliane Regina Pereira do Nascimento ${ }^{3}$, José Luis Guedes dos Santos ${ }^{4}$

Objetivo: esta é uma pesquisa qualitativa que buscou compreender as representações do cuidado de pessoas no limiar da vida. Método: foi realizado com profissionais de saúde de uma Unidade de Terapia Intensiva (UTI) móvel. Os dados foram coletados com entrevistas a 18 participantes (enfermeiros e médicos) e analisados conforme etapas da codificação da Teoria Fundamentada nos Dados. Resultados: o tema central identificado foi: representações do cuidado no limiar da vida, com duas categorias: sentido do cuidado no limite da vida e UTI móvel como espaço do cuidar. Conclusão: o cuidado no limiar da vida precisa ser vivido em seu sentido mais amplo e integral pelos profissionais de saúde.

Descritores: Enfermagem, Cuidados Intensivos, Unidade de Terapia Intensiva.

\section{REPRESENTATIONS OF THE CARE ON THE THRESHOLD OF LIFE BY HEALTH CARE PROFESSIONALS IN MOBILE INTENSIVE CARE UNIT.} Objective: this is a qualitative study with the aim to comprehend care representations for people on the threshold of life. Method: it was conducted with health professionals of the mobile Intensive Care Unit (ICU) mobile. The data was collected through interviews with eighteen participants (nurses and physicians) and the data was analyzed using the codification steps from Grounded Theory. Results: the central theme obtained was: Care Representations on the threshold of life, with two categories: the sense of care on the threshold of life and the Mobile ICU as a space for care. Conclusion: care on the threshold of life needs to be lived in its broadest sense and whole by health professionals.

Descriptors: Nursing, Intensive Care, Intensive Care Unit.

\section{REPRESENTACIONES DEL CUIDADO EN EL UMBRAL DE LA VIDA POR LOS PROFESIONALES DE LA SALUD QUE TRABAJAN EN UNA UNIDAD DE TERAPIA INTENSIVA MÓVIL.}

Objetive: se trata de Investigación cualitativa que tuve como objetivo de comprender las representaciones del cuidado a las personas en el umbral de la vida. Método: se llevó a cabo con los profesionales de la salud que trabajan en una Unidad de Terapia Intensiva móvil. Se entrevistaron dieciocho profesionales (enfermeras y médicos) y los datos se analizaron según las etapas de codificación de la Teoría Fundamentada. Resultados: el tema central identificado fue: las representaciones del cuidado en el umbral de la vida, con dos categorias: el sentido del cuidado en el umbral de la vida y la UCI móvil como un espacio del cuidado. Conclusión: cuidado en el umbral de la vida tiene que ser vivida en su sentido más amplio y completo por profesionales de la salud.

Descriptores: Enfermeria, Cuidados Intensivos, Unidad de Cuidados Intensivos.

${ }^{1}$ Enfermeira. Doutora em Enfermagem. Professora Adjunta da Universidade Federal de Santa Catarina, Florianópolis, SC, Brasil. Email: keyla_nascimento@hotmail.com. ${ }^{2}$ Enfermeira. Doutora em Filosofia da Enfermagem. Professora Titular Universidade Federal de Santa Catarina, Florianópolis, SC, Brasil.

${ }^{3}$ Enfermeira. Doutora em Enfermagem. Professora Associada Universidade Federal de Santa Catarina, Florianópolis, SC, Brasil. ${ }^{4}$ Enfermeiro. Doutor em Enfermagem. Professor Adjunto da Universidade Federal de Santa Catarina, Florianópolis, SC, Brasil. 


\section{INTRODUÇÃO}

O trabalho em Unidade de Terapia Intensiva (UTI) fixa/ móvel visa uma assistência especializada ininterrupta às pessoas em estado crítico, com risco de [perder a] vida. Esse ambiente é marcado por características próprias, como: convivência diária dos profissionais e dos sujeitos com as situações de risco; ênfase no conhecimento técnicocientífico e na tecnologia para o atendimento biológico: constante presença da morte; ansiedade, tanto dos sujeitos hospitalizados quanto dos familiares e profissionais de saúde; e rapidez de ação no atendimento. Isso justifica a introdução de tecnologias por meio de "cuidado tecnológico" para preservar e manter a vida do cliente em estado crítico, o que demanda dos profissionais de saúde conhecimento especializado para interpretar dados objetivos e subjetivos do cliente, e objetivos provenientes do uso do maquinário(1).

Essas características têm contribuído para que a assistência de Enfermagem nessa unidade seja norteada pelo modelo biomédico de assistência à saúde. Diante de insatisfações com esse modo de cuidar em UTI, nas décadas de 1970 e 1980, assevera-se que deve ser considerada a existência de outras necessidades tão importantes quanto aquelas pertinentes à esfera física, quando da implementação de cuidados ${ }^{(2,3)}$.

Desse modo, o cuidar desvela-se para aquele cuidado além da competência técnica(3) para o cuidado humano, para

o cuidado complexo e suscita os questionamentos: qual o cuidado ideal para estas pessoas? Em que o cuidado fomenta a esperança: cura ou conforto (ou seriam os dois)? Como aproximar o técnico e o humano? Essas são questões para vários estudos, mas neste momento busca-se apreender, deste profissional que cuida do ser humano que está no limite da vida, que representações de cuidado ele possui a partir da sua prática profissional

O entendimento dado pelas representações é de que o conhecimento é construido na interação entre indivíduos de um determinado grupo entre si e com o meio. As representações podem ser 'acomodadas/moderadas' ou reelaboradas, dependendo da trajetória pessoal, de sua experiência profissional e do serviço de saúde no qual trabalha. Representações estão nas práticas e são explicitadas em ações e palavras em seus cotidianos de trabalho(4). Assim, as representações são coletivamente construidas e se elaboram das interações sociais, num determinado tempo, cultura e espaço. Diante do exposto, definiu-se como questão de pesquisa: quais as representações do cuidado de pessoas no limiar da vida que os profissionais de saúde que trabalham em uma Unidade de Terapia Intensiva (UTI) móvel possuem?

Este estudo teve como objetivo compreender as representações do cuidado de pessoas no limiar da vida pelos profissionais de saúde que trabalham em uma Unidade de Terapia Intensiva (UTI) móvel

\section{METODOLOGIA}

Pesquisa qualitativa centrada na busca da compreensão das representações que os participantes possuem sobre o objeto do cuidado em sua prática profissional.

O local do estudo foi o Serviço de Atendimento Móvel de Urgência (SAMU) do Estado de Santa Catarina da macrorregião grande Florianópolis. Os participantes do estudo foram 18 profissionais de saúde (04 médicos e 14 enfermeiros) do SAMU, selecionados de acordo com os seguintes critérios: estar graduado há dois anos e possuir experiência profissional em UTI móvel de um ano. Para coleta de dados, utilizouse a técnica de entrevista, a qual foi constituida, inicialmente, de itens referentes à caracterização dos participantes, seguida por questões formuladas em consonância com os objetivos da pesquisa e o questionamento inicial: o que significa pra você cuidar de pessoas em risco de vida?

Para a análise dos dados, utilizou-se a proposta analítica da Teoria Fundamentada dos Dados, por meio da interação com os dados, codificação aberta, codificação axial e codificação seletiva ${ }^{(5)}$. Desse modo, fez-se uma análise vertical (em cada entrevista) e horizontal (conjunto das entrevistas), de forma a encontrar convergências e divergências, no processo de codificação aberta. Em seguida, na codificação axial, os dados foram agrupados por meio das conexões entre as categorias. Da codificação seletiva, emergiu o tema central, ou seja, uma categoria que expressa o principal tema ao redor do qual as categorias giram ${ }^{(5)}$

Para compreender e apreender o cuidado no limiar da vida, buscou-se o suporte teórico da Teoria do Limite proposto 
por Eugênio Trías, bem como o referencial de complexidade/ sistemas. A Teoria do Limite possibilita a reflexão sobre os limites que acompanham o desenvolvimento e aplicação das tecnologias e ciências para o bem-estar e respeito no cuidado aos seres humanos ${ }^{(6)}$. Na perspectiva da complexidade, o cuidado é uma rede ou teia de processos e produtos que envolvem relações, interações e associações entre os seres, sendo parte organizador do sistema de saúde e parte organizador do sistema de cuidado(7).

Foram respeitados os princípios éticos e legais da pesquisa envolvendo seres humanos. O estudo foi aprovado pelo Comitê de Ética em Pesquisa de referência. Para a identificação dos sujeitos, elaborou-se uma escala de convenção: $M$ - médico, E - enfermeiro/a - seguido da ordem das entrevistas: 01, 02, ...18. Exemplo: M04.

\section{RESULTADOS E DISCUSSÃO}

Foi identificado o tema central: Representações do cuidado no limiar da vida, com duas categorias: "O sentido do cuidado no limite da vida" e "A UTI móvel como espaço do cuidar".

A categoria: O sentido do cuidado no limite da vida, na forma como os profissionais percepcionam o que é o cuidado no limite da vida originou três subcategorias: Universo reificado das representações do cuidar; Universo consensual do cuidar em sua dimensão técnico-prática e Universo relacional.

A subcategoria Universo reificado das representações do cuidar refere-se às informações, ao ensino, ao conhecimento adquirido e aos programas de educação permanente que permitem a circulação dos conhecimentos científicos entre os profissionais. Os participantes destacaram a importância do conhecimento quando mencionaram que "o teu conhecimento, aquilo que tu aprendeste, as pessoas que estão contigo, é isso que vai decidir"(ElO). "[...] não pode deixar de estudar, tem que ser sempre uma pessoa esforçada, no sentido de estar estudando [...]" (M16).

Articular e organizar os conhecimentos e, assim. reconhecer e conhecer os problemas do mundo, torna-se desejável para a reforma do pensamento(b). É importante compreender que o conhecimento não progride por formulação, sofisticação ou abstração, mas progride principalmente pela capacidade de contextualizar ${ }^{(7,8)}$.

O cuidado deve estar pautado no universo retificado do conhecimento e este pode ser ampliado à medida que se realiza ações de educação em serviço. Desse modo, é necessário proporcionar ao cuidador capacitações para o desempenho de suas atividades que visem aprimorar a capacidade do ser para o envolvimento no cuidado com o outro. "O conhecimento muda [...] cada situação é uma situação, que deveria ser discutida, estudada, deveria ser feito protocolo". (M15)

Na UTI móvel, bem como em outras unidades, torna-se desejável que os profissionais disponham de curiosidades e competências éticas consistentes, em função da missão que thes é confiada pela sociedade: o cuidado no limiar da vida: "Aprender! Sempre aprender. Então, deixa eu filosofar: "Mestre não é quem ensina, mestre é quem aprende" [...] vou aprender e quero continuar aprendendo até parar de fazer Medicina" (M04).

O cuidado intensivo requer compromisso, responsabilidade, dedicação e conhecimento, de modo a estabilizar uma situação clínica e a preservar a vida.

A subcategoria: Universo consensual do cuidar em sua dimensão técnico-prática sustenta que além do saber, é igualmente exigido aos profissionais o saber-fazer, ou seja, o cuidado é visto como a ação de realizar uma atividade, atuar junto a um agente, executar algo para ou junto ao outro ser, agir em benefício da saúde do outro: "[...] ser rápido, ser eficiente naquele procedimento porque senão a gente está colocando em risco a vida daquele paciente" (EO5).

Assim, o cuidar é uma atividade realizada no cotidiano organizacional de gerir e assistir. Esse processo é cíclico, no qual o profissional deve priorizar e aprimorar relações, especialmente a partir de atitudes como estar com, tomar conta de, entre outros: "Cuidado é fazer aquilo que o outro não pode fazer por si" (EO8).

E, a subcategoria: Universo relacional que traz a dimensão relacional de cuidado desvela o entendimento de que os relacionamentos humanos expressam-se por uma relação/ interação empática: "Cuidado pra mim é me preocupar com outra pessoa" (ElO). "Uma coisa que eu me preocupo muito é olhar pra pessoa, falar o que está sendo feito" (EOI).

O envolvimento relacional é como uma postura interna de voltar-se para o outro, no qual a reciprocidade e a autenticidade são elementos básicos nessa relação(9). Os participantes também relacionam o cuidado com 0 envolvimento relacional: "O conhecimento não só técnico como o humano também, de respeito, de consideração, de fazer uma assistência ideal (EO2). "Cuidado traz muito a palaura compreensão, humanidade, respeito. O respeito à outra pessoa, à situação a que ela se encontra" (M11).

O estar com o outro, no eixo relacional de cuidado, requer aproximação, sem receitas modeladoras. Porém, para haver uma relação no cuidado, o profissional precisa desenvolver a capacidade de aproximação e observação do ser cuidado em todas as dimensões: "[...] o sentimento de ajuda é essencial nesse cuidado, numa perspectiva empática, colocamonos no lugar do outro..." (El2). Os profissionais de saúde, em especial os enfermeiros, vêm buscando progressivamente 
uma assistência humanística, centrada no cuidado ao ser humano: "Étu ter um cuidado humanizado, olhar o serhumano como um todo. Não adianta cuidar só da mente, cuidar só do corpo, ver as pessoas como se fossem partes" (El4).

O foco no cuidado intensivo é a resolutividade e o direcionamento das ações para promoção do bem-estar do paciente crítico visando ao preenchimento das necessidades humanas básicas ${ }^{(10)}$. Nesse sentido, a ênfase do trabalho é dirigido às necessidades do indivíduo, tendo que, por conseguinte, possuir sólidos conhecimentos científicos, técnicos e a sensibilidade humana. Um dos participantes salienta que: "O cuidado resume-se a uma aliança entre amor, carinho e conhecimento técnico-científico. Do carinho pelo outro ser humano que está numa situação de total dependência de você naquele momento. Do amor, acho que todo ato de ajudar, no sentido de amenizar, confortar, acalmar, tirar a dor, consolar ou até mesmo o ato de cerrar as pálpebras do paciente, é sempre um ato de amor." (MI6).

Essa fala reflete o eixo do sentido do cuidado no limite da vida, pois os profissionais necessitam de conhecimentos que irão nortear a assistência durante $\mathrm{o}$ atendimento ao paciente grave em UTImóvel. O saber incorporado às práticas torna-se imprescindivel, bem como a aproximação dos sujeitos que, por sua vez, favorece 0 cuidado nesse ambiente de trabalho.

$$
\text { A categoria UTI }
$$

móvel como espaço do

cuidar,evidencia que o espaço do sistema de cuidados é marcado pelas vidas vividas, pelas oportunidades espaciais das relações de trabalho e pelo vivenciar o cuidado na ordem do espaço, dependência, pertencimento e privacidade. É o lugar pontilhado por uma multiplicidade de pequenos espaços de provimento de saúde, da busca de melhoria do corpo num coletivo de afeto, emoções/ambiência e determinações ${ }^{(11)}$. Os relatos retratam um novo olhar acerca do local em que o paciente se encontra, ou seja, é estar cada dia em um local novo, diferente da terapia intensiva na qual há uma definição de pacientes: "A UTI móvel tem características distintas, porque você está se deslocando e vai chegar num ambiente que você não conhece [...]" (MI6). "Na UTI móvel tudo é uma caixinha de surpresas[...]"(M15).

Há diferença entre cuidar de pacientes em UTI móvel e cuidar em UTI no contexto hospitalar quanto à área física, aos recursos disponiveis e ao número de profissionais de saúde para o atendimento: "Dentro da ambulância, a gente tem o que tem na UTI, mas a gente tem limitação dos espaços" (M07). Entretanto, percebe-se uma semelhança com a terapia intensiva pelo fato do paciente necessitar de cuidados intensivos: "Mas UTI é igual a do hospital no sentido de salvar o paciente no risco de vida" (EO5). Desse modo, o cuidado intensivo baseia-se no tripé: paciente grave, equipamento altamente técnico e equipe multiprofissional especializada com conhecimentos e experiência para cuidar do paciente.

É importante entender o contexto do ambiente de trabalho desses profissionais: um veículo ambulância. Tal ambiente encontra-se em movimento e, em caso de intercorrências, deve parar para que se realize algum procedimento. $\bigcirc$ espaço interno é restrito, os assentos dos profissionais de saúde são posicionados de frente para a maca e o corredor é estreito, o que limita a movimentação, mas permite que os equipamentos e materiais fiquem sempre à disposição nos casos de urgência. Os profissionais enfrentam situações imprevisiveis, tais como, falhas com os equipamentos, percurso de ambulância em estrada ruim e locais de difícil acesso: "[...]O veiculo está em movimento e você vai ter que parar numa via pública para fazer um atendimento, ou para fazer uma medicação porque o paciente está agitado. Tudo é ruim [...]" (EO9).

O trabalho em um ambiente como esse pode levar a equipe de saúde ao desgaste físico e emocional. O constante estresse a que esses profissionais são submetidos pode gerar repercussões na saúde, principalmente em relação ao padrão de sono e prejuízos à memória e capacidade de concentração. Portanto, profissionais desses setores são um grupo de trabalhadores que precisa ser cuidado ${ }^{(12)}$.

Para a eficiência e eficácia do cuidado, é importante que haja sincronia e coesão no cuidar, manutenção adequada dos equipamentos e relações efetivas entre as pessoas da equipe. Esses são fatores que impulsionam o trabalho a ter um sentido diferente, deixando de ser visto como sofrimento, e passando a ser um local interessante, que denota satisfação profissional: "Quando a gente trabalha nesse serviço, trabalha porque gosta.[...] tem uma possibilidade de vencer esses desafios e fazer alguma coisa por alguém, apesar das adversidades" (M15). "Vamos para a ambulância, porque 
gostamos daquilo que a gente faz!" (EO8).

As experiências vividas pelos profissionais em UTI móvel perpassam a rotina mecanicista/biologicista do dia a dia, mas alcançam o cuidar humanizado/sensivel, denotando a abordagem integral do ser humano em todas as dimensões ${ }^{(6,7)}$. Dessa forma, nesse ambiente de cuidado fluem sentimentos de ansiedade, tristeza e alegria expressados de maneira positiva ou negativa.

Portanto, o cuidado no limiar da vida em UTI móvel acontece por meio de um processo coletivo baseado no saber-fazer-agir de cada profissional e permeado por relações, interações e associações múltiplas e complexas. Esse contexto desperta entre os profissionais sentimentos contraditórios diante dos limites vivenciados e impostos por situações e circunstâncias nas quais o cuidado a individuos que se encontram em risco de morte é realizado.

\section{CONCLUSÃO}

Este estudo mostrou que os profissionais de saúde de uma UTI móvel possuem representações permeadas de subjetividades a respeito do cuidado praticado em seu trabalho, evidenciado no tema central: Representações do cuidado no limiar da vida, resultado da intercepção de duas categorias: Sentido do cuidado no limite da vida e UTI móvel como espaço do cuidar.

$\mathrm{Na}$ primeira categoria, foram identificadas três subcategorias que revelam como o cuidar é concebido, vividoe caracterizado pelos participantes da pesquisa. Encontraramse asserções em três vertentes, nas quais o cuidado é visto como científico, técnico e relacional. No conjunto dessas três vertentes, traduz-se o cuidado no limiar da vida e este precisa ser vivido em seu sentido mais amplo e integral pelos enfermeiros e médicos.

Na segunda categoria, constatou-se que os participantes percebem a UTI móvel com muitas particularidades. Porém o cuidado/assistência por eles prestado tem suas raízes na terapia intensiva. Isso se deve em grande parte ao fato de que os pacientes atendidos são críticos e demandam uma assistência direta e dependente.

Os momentos críticos vivenciados pelos profissionais estão permeados por sentimentos não exteriorizados no dia a dia, em um trabalho cansativo e desgastante em que se lida o tempo todo com o imprevisível, o que gera, muitas vezes, a sensação de impotência diante das situações estressantes. Assim, a UTI móvel no cuidado de pacientes no limite da vida ainda é um desafio constante no cenário do cuidado à saúde, o qual requer novas investigações futuras.

\section{REFERÊNCIAS}

1 Silva RC, Ferreira MA, Apostolidis T. Estilos de cuidar de enfermeiras na Terapia Intensiva mediados pela tecnologia. Rev. Bras. Enferm. 2014; 67(2): 252-60.

2. Waldow VR. Cuidado colaborativo em instituições de saúde: a enfermeira como integradora. Texto Contexto - Enferm. 2014:23(4):1145-52.

3 Watson J. Enfermagem: ciência humana e cuidar uma teoria de enfermagem. Rio de Janeiro: Lusociência; 2002.

4 Giami, A.; Veil, C. Enfermeiras frente a Aids: representações e condutas, permanências e mudanças. Canoas: Ulbra, 1997.

5. Corbin J,Strauss A Basics of qualitative research: techniques and procedures for developing grounded theory. Thousand Oaks, CA: SAGE Publications; 2014

6 Nascimento, KC; Erdmann, AL. Reflexões sobre a filosofia do limite e suas implicações para o cuidar em enfermagem. Aquichan. 2009: 9(2):185-92.

7. Erdmann AL, Mello ALSF, Meirelles BHS, Marino SRA. As organizações de saúde na perspectiva da complexidade dos sistemas de cuidado. Rev. Bras. Enferm. 2004 ; 57(4):467-71.

8 Piexak DR, Backes DS, Santos SSC. Cuidado de enfermagem para enfermeiros docentes na perspectiva da complexidade. Rev. Gaúcha Enferm. 2013:34(2):46-53.

9 Nascimento KC, Erdmann AL. Understanding the dimensions of intensive care: transpersonal caring and complexity theories. Rev. Latino-Am. Enfermagem. 2009;17(2):215-21.

10 Almeida AS, Barlem ELD, Barlem JGT, Lunardi VL. Produção científica da enfermagem sobre morte/morrer em unidades de terapia intensiva. Enferm em Foco. 2013; 4(3,4):179-83.

11 Baggio MA, Erdmann AL. Processando o cuidado "do nós" nas relações/ interações estabelecidas por profissionais de enfermagem e de saúde. Cogitare Enferm. 2015; 20(3): 573-80.

12 Salvador RSP, Silva BASA, Lisboa MTL. Estresse da equipe de enfermagem do corpo de bombeiros no Atendimento Pré-Hospitalar Móvel. Esc. Anna Nery. 2013;17(2):361-8. 